\title{
New Artificial Immune System Approach Based on Monoclonal Principle for Job Recommendation
}

\author{
Shaha Al-Otaibi \\ College of Computer and Information Sciences \\ Princess Nora bint Abdulrahman University \\ Riyadh, Saudi Arabia
}

\author{
Mourad Ykhlef \\ College of Computer and Information Sciences \\ King Saud University \\ Riyadh, Saudi Arabia
}

\begin{abstract}
Finding the best solution for an optimization problem is a tedious task, specifically in the presence of enormously represented features. When we handle a problem such as job recommendations that have a diversity of their features, we should rely to metaheuristics. For example, the Artificial Immune System which is a novel computational intelligence paradigm achieving diversification and exploration of the search space as well as exploitation of the good solutions were reached in reasonable time. Unfortunately, in problems with diversity nature such job recommendation, it produces a huge number of antibodies that causes a large number of matching processes affect the system efficiency. To leverage this issue, we present a new intelligence algorithm inspired by immunology based on monoclonal antibodies production principle that, up to our knowledge, has never applied in science and engineering problems. The proposed algorithm recommends ranked list of best applicants for a certain job. We discussed the design issues, as well as the immune system processes that should be applied to the problem. Finally, the experiments are conducted that shown an excellence of our approach.
\end{abstract}

Keywords-content-based filtering; computational intelligence; artificial immune system; clonal selection; monoclonal antibodies

\section{INTRODUCTION}

The Job Recommender System (JRS) has emerged in ebusiness online services in recent years. While companies post their jobs on online portals, an applicant uses them to establish his/her profile. A huge volume of job descriptions and applicant profiles are becoming available online. The need increases for applying the recommender system technologies that can support recruiters to handle the huge online information efficiently [1], [2].

Additionally, we notice the diverse nature of job specification that should be considered in candidates/job matching. Certain job requirements should be modeled in many forms to meet a diverse set of candidates that satisfy job requirements. We need to determine the job's requirements by a set of vectors of different features that can meet most possible appropriate applicants, and then rank applicants depending on the matching degree between the applicant and job requirements. We proposed an algorithm for JRS that used the traditional Artificial Immune System (AIS) paradigm [3].

The antibodies production in AIS algorithm used the natural response of the adaptive immune system that similar to the production of Polyclonal Antibodies (PAbs) in laboratories where the population of antibodies produced from multiple B- cells that have been activated by the immune response. The immune response to an antigen generally involves the activation of multiple B-cells all of which target a specific antigen. Consequently, a huge number of antibodies are generated with different affinities for that antigen. The antibodies and antigens in AIS algorithm represented by a vector composed of set of features that represents the job and applicant profiles.

Unfortunately, when we handle a problem such as job recommendations with a diverse nature in their features, the traditional AIS produced a huge number of antibodies that affects the system efficiency. Hence, The AIS applied the mutation strategy to perform the diversity topic in the previous algorithm. To leverage this issue, we will apply the AIS with Monoclonal Antibodies (MAbs) production principle that produces a population of antibodies from a single B-cell that recognize the antigen. However, the discovery of monoclonal antibodies has encouraged a revolution in medicine that is probably only second to the discovery of vaccination [4].

The algorithm that we will propose in this article is named Monoclonal Artificial Immune System for Job Recommender System (MCAIS-JRS). It recommends ranked list of best applicants for a certain job. We will represent each job's feature by a single antibody that tries to recognize the epitope of the antigen (applicant's feature) instead of using single antibody to represent a set of features. Hence, certain antigen has many epitopes surfaces that the antibody tries to recognize a single epitope at a time. Additionally, we propose a diversity operator to be used instead of mutation, and maintain a diversity pool of alternative solutions. Although, the proposed algorithm covers a wider range of problems that have numerous features with different alternatives such our considered problem.

This article is organized as follows: Section II demonstrates the related work. Section III presents the artificial immune system concepts used as basic technique in our proposed algorithm. Section IV illustrates the different issues related to modeling job recommendation in AIS using monoclonal antibodies principle. For example, the representation of both antibody and antigen, the applying of the diversity topic, as well as the using of similarity measures. Section V details the description of the proposed algorithm. Section VI shows the experimental results and the scalability study. Finally, we conclude our findings of this article in Section IVI. 


\section{RELATED WORK}

In recent years, much research has been conducted to discuss different issues related to the applying of recommender system technologies in job problem [5]. A survey of job recommender system was presented [6]. It covered the job requirements such as user profiling and similarity measures. Additionally, we presented a comprehensive survey of job recommendation and listed the advantages and disadvantages of technical approaches in different job recommender systems [5]. Moreover, author of [7] determined that we must consider unary attributes such as individual skills, mental abilities and personality that control the fit between the individual and the tasks to be accomplished. Several recommender system techniques have been applied in job/candidates matching problem, started by the personnel selection approach [1] that developed a probabilistic hybrid recommendation approach for job/candidates matching. Then, their model utilized and extended by [7], [8], and [9]. The model that tries to use the suitable personality traits and key specialized skills through information statistics and analytic hierarchy process was presented [10], [11].

Fazel-Zarandi and Fox combined different matchmaking strategies in a hybrid approach for matching job seekers and jobs using logic-based and similarity-based matching [12]. Additionally, the PROSPECT, which is a decision support tool assisting recruiters to shortlist candidate resumes list. It mines resumes to extract features of candidate profiles such as skills, education, and experience. It used IR techniques to rank applicants for a given job position [13]. The recommendation problem treated as a supervised machine learning problem [14]. This system recommends jobs to applicants based on their past job histories, in order to facilitate the process of choosing a new job. It trains a machine learning model using a large amount of job transitions extracted from person profiles available in the web.

Moreover, the TalentMatch system used at LinkedIn, in which the recommender system activated by a job posted on the site, scans the entire database to find the best candidates for the job. In this system, the semantic model computes the probability that the feature vector representing the candidate and the feature vector representing the job are a good match [15]. The hybrid recommender system that uses the job and user profiles, as well as the activities undertaken by users, in order to produce personalized recommendations of candidates and jobs. The generated data is modeled using a directed, weighted, and multi-relational graph, and the ranking algorithm [16]. Finally, candidate's profile and candidate's job preferences have been used to predict job recommendations. First, rules predicting the general preferences of the different user groups are mined. Then, the job recommendations for target candidate are made based on content-based filtering as well as candidate preferences, which are conserved either in the form of mined rules or obtained by candidate's own applied jobs history [17].

\section{ARTIFICIAL IMMUNE SYSTEM}

The immune system has started to the emergence of AIS as a novel computational intelligence paradigm in the 1990s. A number of AIS models were existent, and they are used in pattern recognition, optimization, computer security, fault detection, and many other applications [18]. The AIS can be described as metaphorical computational systems developed using ideas, theories, components, and process derived from the immune system. It has two parts, the innate and adaptive immune systems [19]. The innate immune system is a stable mechanism that perceives and destroys specific invading organisms, whereas the adaptive immune system responds to anonymous foreign invader and provides a response that can persevere in the body over a long period of time. The adaptive immune system is comprised of a collection of different cells accomplishing different functions that spread over the body. The B-cells are the primary cells that responsible for the generation and secretion of antibodies using specific proteins which binding the antigen. Each B-cell can only provide one particular antibody. The antigen is located on the surface of the invading organism, and the binding of an antibody to the antigen is a signal to kill the invading cell [19], [20].

In general, there are many theories for the immune system, we will focus in this article on the clonal selection and mutation strategy that are primarily used in AIS models. The clonal selection is an algorithm used to define the basic features of an immune response to an antigenic stimulus. It is defined the idea that only those cells that recognize the antigen are proliferate. The mutation process is the most commonly used in AIS models; it means random genetic changes to the genes of the cloned cells. These changes caused proliferation and variation of high affinities antibodies. This concept was used as the basis for mutation in clonal selection algorithm, where the mechanism was the affinity between the antibody and the antigen. Additionally, the mechanism is often enhanced by the somatic hypermutation that is noticed as a process for optimizing the binding affinity of antibodies [21].

Moreover, there are two ways to produce antibodies in laboratories. Hence, antibodies include those produced by a combination of various B-cells, termed polyclonal antibodies and those secreted by a single cell of B-cell, termed monoclonal antibodies; both types have become crucial instruments in fundamental immunological research, diagnostic testing, immunohistochemistry, and vaccine quality control. PAbs and MAbs can be used for these purposes, although the production of these antibodies requires the use of a large number of animals. PAbs represent a population of antibodies collected from multiple B-cells that have been activated by the immune response of an immunized animal.

The immune response to an antigen includes the activation of multiple B-cells all of which target a specific epitope on that antigen. As a result, a huge number of antibodies are generated with different epitope affinities. However, MAbs represent a population of antibodies that recognizes a single epitope within an antigen, and produced by a single B-cell.

Authors of [22] discovered that this cell can be immortalized by fusion with myeloma cells, resulting in set of cells that are able to produce unlimited quantities of monoclonal antibodies. Since the Nobel prize winning work of these researchers, MAbs have become essential treatment in basic research as well as in diagnostic testing and medical 
treatments. The PAbs and MAbs production processes are illustrated in Fig. 1 that adapted from Biotech Resources ${ }^{1}$.

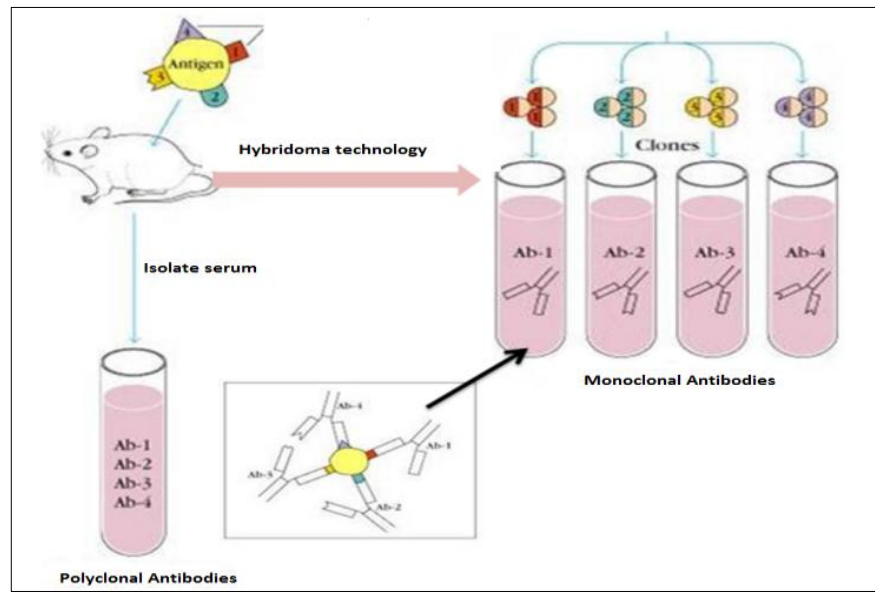

Fig. 1. The procedure of PAbs and MAbs production

Using the MAbs production principle that produces a population of antibodies from a single B-cell to recognize the antigen will leverage the previously mentioned issue in the traditional AIS [3]. However, the antibodies and antigens in traditional AIS algorithm represented by a vector composed of set of features that represents the job and applicant profiles. Unfortunately, when we handle a problem such as job recommendations that has too many features with a diversity in their nature, the traditional AIS produced a huge number of antibodies that affects the system efficiency. Hence, the AIS applied the mutation strategy to perform the diversity topic in the previous algorithm.

\section{MODELING Job RECOMMENDATION IN AIS USING MONOCLONAL ANTIBODIES PRODUCTION PRINCIPLE}

\section{A. Antibody and Antigen Representations}

Choosing a suitable representation is very important for the algorithm's success. In JRS, there are many features that should be represented to model the job's requirements as well as the applicant's resume. For example, a set of qualifications and their information, skills, languages, experience, etc. The antibodies will represent certain job's profile. While, the antigens represent all applicants' profiles in the system. In MCAIS-JRS, an antibody indicates a specific feature that tries to recognize single epitope of the antigen. Hence, the antigen has many epitopes surfaces that the antibody tries to recognize a single epitope at a time. A possible mapping of the antibody's representation to vector space is

(<AB-CODE $\rangle,\langle$ AB-FEATURE $>,\langle$ AB-STRENGTH $\rangle$, $<$ AB-FEATURETYPE $>$ )

where $\angle \mathrm{AB}-\mathrm{CODE}>$ gives a certain code for the current antibody, $\angle A B-F E A T U R E>$ is value of a certain job's feature, $<$ AB-STRENGTH $>$ represents the antibody strength, it counts how many certain job's feature recognized. Hence, each recognized feature would increase the strength operator for current antibody. Finally, the <AB-FEATURETYPE > uses to

\footnotetext{
${ }^{1}$ http://www.bio.davidson.edu/molecular/MolStudents/01rakarnik/mab.html
}

distinguish a certain feature from other features when comparing it with antigen's features.

Example 1 Let us consider the following antibody's vector: $(3,2,23,7)$.

where,

3 is the $\langle\mathrm{AB}-\mathrm{CODE}\rangle$,

2 is the $\angle A B-F E A T U R E>$ that refers to the Electrical Engineering specialty,

23 is the <AB-STRENGTH>, it means the Electrical Engineering specialty found in 23 applicants' profiles, and

7 is the <AB-FEATURETYPE>, where 7 represents the $<$ MSC SPECIALTY >, that means this antibody will be compared only with MSC specialty feature for the antigens.

On the other hand, the antigen represented by a vector space that contains a set of features. Each feature treated as an epitope that the antibody tries to recognize. A possible antigen's representation is:

(<APPLICANT-ID $>$,

$(<$ PHD DEGREE $><$ PHD GPA $><$ PHD SPECIALTY $>)$,

$(<$ MSC DEGREE $><$ MSC GPA $><$ MSC SPECIALTY $>)$,

$(<$ BSC DEGREE $><$ BSC GPA $><$ BSC SPECIALTY $>)$,

$(<$ DIPLOMA DEGREE $><$ DIPLOMAGPA $><$ DIPLOMA

SPECIALTY $>$,

$(<$ LANGUAGE $><$ LANGUAGE LEVEL $>)$,

$\langle$ JOB CATRGORY>, 〈TYPE OF SKILL〉, 〈EXPERIENCE〉, $<$ AFFINITY>).

Example 2 Let us consider the following applicant's vector,

$(1,(0,0,0),(1,4.1,2),(1,4.02,2),(0,0,0),(1,3), 1,5,0)$.

where applicant id $=1$,

$(0,0,0)$ : no $\mathrm{PhD}$ degree,

$(1,4.1,2)$ : MSc degree with 4.1 GPA and spatiality number 2 (2 refers to Electrical Engineering),

$(1,4.02,2)$ : BSc degree with 4.02 GPA in Electrical Engineering,

$(0,0,0)$ : no diploma degree,

$(1,3)$ : 1 refers to English language and 3 refers to excellent level,

1 refers to the OO programming skills,

5 refers to Experience years,

0 refers to the Affinity that represents the total distance between the current job (represented by a set of antibodies $\left.A b_{i}\right)$ and any applicant $\left(\mathrm{Ag}_{\mathrm{i}}\right)$. We will discuss how to calculate the total similarity in the following subsection.

\section{B. Similarities Measures}

The similarity or affinity is an important issue in building any AIS algorithm. The affinity is taken as the distance between a given antigen and the antibody. In MCAIS-JRS, we used mixed representations. Subsequently, the affinity is calculated using a combination of different types of similarity measures depending on the features types. Additionally, some of the parameters needs multi-level checking. It needs to determine if it is required by the recruiter for the recent job or 
not. For example, the certain degree that the applicant has may be it is not required for a certain job; in such case, this degree should not be scored. Then, the parameters distance is taken as a Boolean match 0, no match 1 as in

$$
D_{K_{j}, i}= \begin{cases}1, & \text { if } K_{j} \neq K_{i} \\ 0, & \text { otherwise }\end{cases}
$$

where $\mathrm{D}_{\mathrm{Kj}, \mathrm{i}}$ represents the similarity between the antigen and antibody for specific parameter $K, j$ represents the current antigen $\left(\mathrm{Ag}_{\mathrm{i}}\right)$ and $i$ represents the current antibody $\left(\mathrm{Ab} \mathrm{b}_{\mathrm{i}}\right)$. For real attributes such as the GPA for certain degree, the similarity is calculated as a normalized distance between a given antigen and antibody.

$$
D_{K_{j}, i}=\frac{-a\left(K_{j}-K_{i}\right)}{K_{\text {MaxValue }}}
$$

Where $K_{\text {MaxValue }}$ is the maximum value for current attribute. For example, if the maximum GPA is 5 then the $K_{\text {MaxValue }}$ is 5 . For example, if the applicant GPA is more than the job's required GPA it will be decreased the similarity (use minus). For integer attributes such as a certain specialty or skill, the similarity is also taken as (1). The total affinity for a certain antigen is calculated as

$$
\operatorname{AFFINITY}_{\mathrm{j}}=\frac{\left(\sum_{\mathrm{k}=0}^{\mathrm{L}-1} \mathrm{D}_{\mathrm{k}}\right)}{\mathrm{I} .}
$$

where $j$ represents the current antigen $\left(\mathrm{Ag}_{\mathrm{i}}\right), K$ is the current parameter and $L$ is total number of parameters. The AFFINITY $_{i}$ takes values between $[-1,1]$, where the similarity between the antibody and the antigen decreased from negative values to positive values. This means the AFFINITY $\mathrm{i}_{\mathrm{i}, \mathrm{i}}=0$ is better than AFFINITY $_{\mathrm{j}, \mathrm{i}}=0.99$.

\section{Diversity Operator}

The mutation process used in most AIS algorithms as main operator such as our previous algorithms in [3]. Although the exploration of search space achieved by using mutation process to reach higher affinity matches the invading antigen. It performs random changes to the cloned cells that are involved in controlling the antigen receptor. These changes caused proliferation and variation of high affinities antibodies. Therefore, a huge number of antibodies are generated with different affinities for that antigen. When, we handle a problem such as job recommendation that has enormous features with multiple alternatives, the mutation process will produce a huge number of antibodies that affects the system efficiency. To handle this issue, we apply the MAbs production in the AIS algorithm as well as the using of Diversity Operator instead of mutation to explore the search space.

The novelty here is that we propose a Diversity Operator to be used instead of mutation, and we maintain a diversity pool of alternative solutions. This works on each feature alone. Although, the purpose of the proposed algorithm is to cover a wider range of problems that have many features with more alternatives such as job recommendation problem. For example, some features such as specialties and skills have many possible choices that can be considered as possible valid solutions, and they will be added to the diversity pool. Consequently, a single antibody will be generated for each feature alternative and will be added to the antibodies pool. Figure 2 displays the antibodies' production in MCAIS-JRS for a certain job's profile and illustrates the Diversity Operator concept.

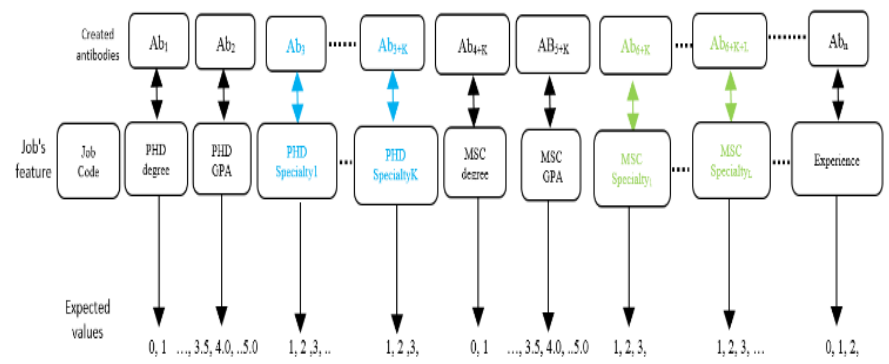

Fig. 2. Antibodies' production for a certain job profile

\section{MONOClONAL ALGORITHM FOR Job ReCOMMENDER SYSTEM}

This section presents the design details of the proposed algorithm. We start by describing the metaphors and the parameters that used in MCAIS-JRS algorithm, where the antibody is a certain job's feature and the antigen is a vector of features for an applicant that considered as a target to be checked.

$\begin{array}{ll}\mathrm{F} & \text { features list. } \\ \mathrm{AB} & \text { antibodies pool. } \\ \mathrm{Ab}_{\mathrm{i}} & \text { current antibody. } \\ \mathrm{n} & \text { number of antibodies. } \\ \mathrm{f}_{\mathrm{k}} & \text { certain feature. } \\ \mathrm{f}_{\mathrm{i}} & \text { current alternative for certain } \mathrm{f}_{\mathrm{k}} . \\ \mathrm{AG} & \text { antigens pool. } \\ \mathrm{Ag}_{\mathrm{j}} & \text { current antigen. } \\ \mathrm{Affinity} & \text { total distance between a given } \mathrm{Ag}_{\mathrm{j}} \text { (applicant) and antibody }\left(\mathrm{Ab}_{\mathrm{i}}\right) . \\ \mathrm{N} & \text { maximum size of antibodies pool. } \\ \text { Threshed } & \text { acceptance level of antigen. } \\ \text { Aga } & \text { set of accepted antigens. }\end{array}$

The MCAIS-JRS starts by initializing some parameters such as the size of the antibodies pool $(\mathrm{N})$ and the acceptance level of the antigen (Threshold). All antigens in the system will be exposed to the MCAIS-JRS algorithm steps in the same way. The algorithm performs one generation once all available antigens have been exposed to the antibodies cells, and all the MCAIS-JRS algorithm steps have been performed for each antigen. Figure 3 displays a diagrammatic representation of the MCAIS-JRS algorithm steps and Table 1 presents the pseudo code of the algorithm. 


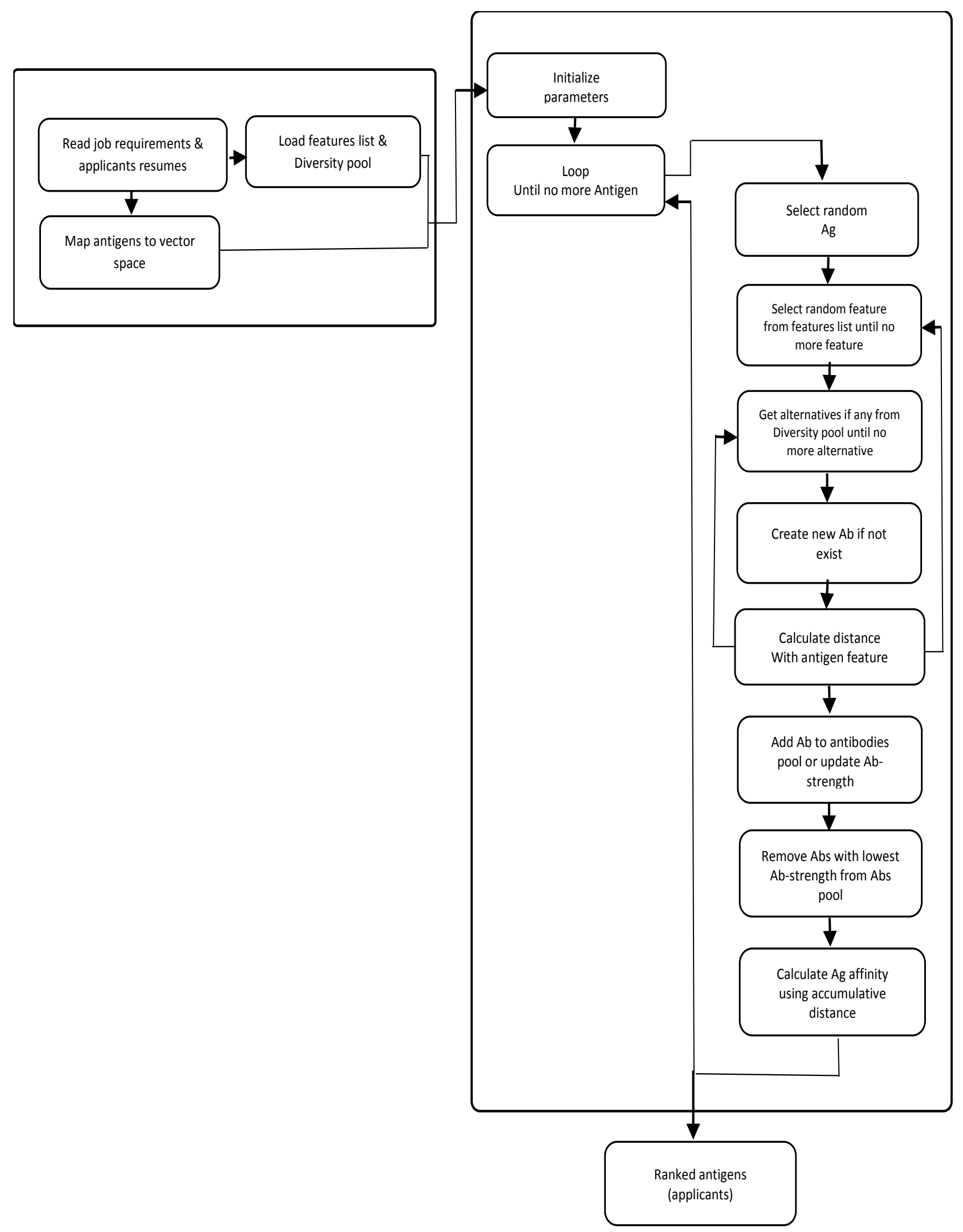

Fig. 3. A diagrammatic representation of the MCAIS-JRS 
The following steps illustrate the MCAIS-JRS:

- The initial population is generated using the applicants' profiles (antigens set) and the job profiles (antibodies set).

- While the stopping condition is not met (no more antigens), the algorithm proceeds by performing a number of iterations to expose all antigens in the system. We use diversity operator to perform the job's diversification (e.g. specialties and skills).

1) The system selects random antigen (applicant), and the distance will be calculated for all antibodies (job's features) against the selected antigen.

2) Select a new feature from the job's features list $(F)$.

3) Check the diversity pool that associated to the current job's profile and take one alternative for the selected feature if any. $\left(A b_{i}\right)$.

4) Create antibody for the selected feature's alternative

5) Each antibody represents a certain job's feature that match the same feature of the antigen (applicant). If match, then increase the $\left\langle A b_{i}\right.$-STRENGTH>. Repeat 3-5 until no more alternatives for current feature.

TABLE I. THE PSEUdo CODE FOR MCAIS-JRs ALgorithm

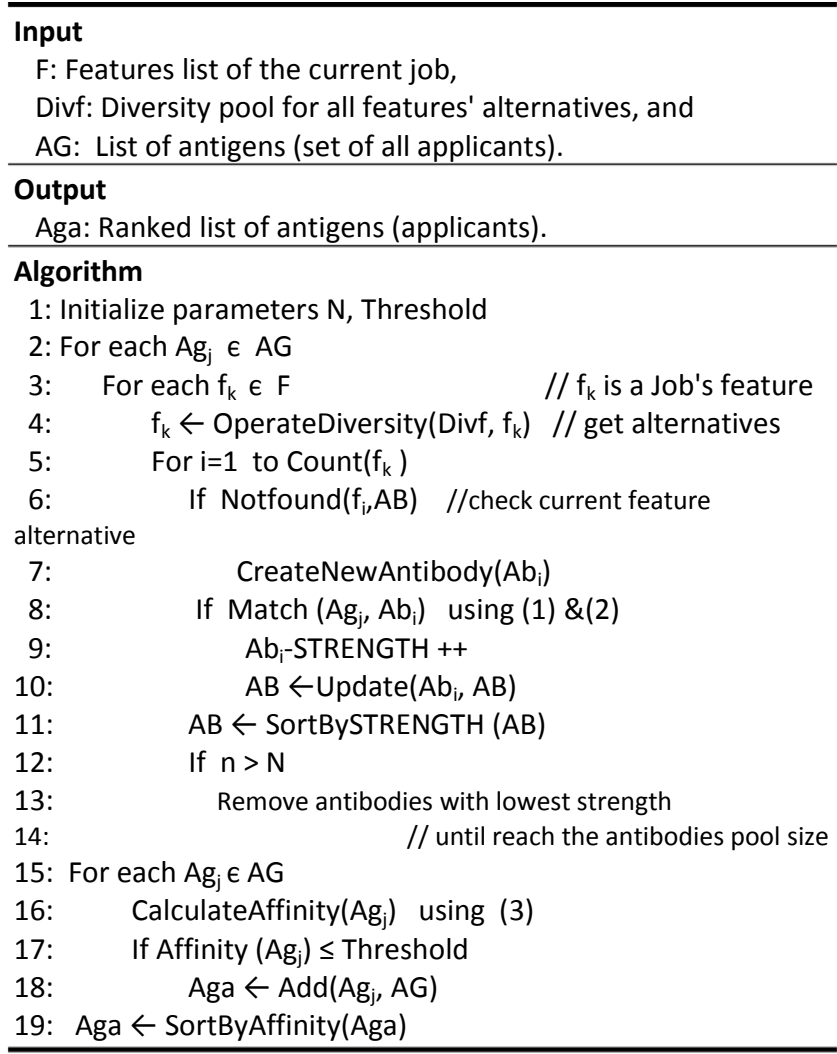

1) Repeat 2-5 until no more features.

2) If the total number of antibodies is more than the maximum size of the antibody's pool $(N)$, the antibodies with lowest strength will be removed.

3) The antigen's affinity will be calculated as accumulative value from the distance with all antibodies. Then, the antigen's affinity is checked against a certain threshold that specified by the job's recruiter to determine the acceptance of an antigen.

4) The antibodies pool then taken to expose next antigen.

- Finally, the set of antigens (applicants) will be ranked depending on their affinities to produce Aga list.

\section{EXPERIMENTAL RESULTS}

The MCAIS-JRS was constructed from the algorithmic outlines given in the previous sections. Therefore, the proposed recommender system integrated the monoclonal antibodies production principle and AIS processes, as well as, a diversity operator that used instead of the mutation strategy. This recommender system focus on the find good items task, where the job's recruiter is provided with a ranked list of qualified applicants who match the job's requirements. We collected data from resumes spread on the internet and generated the missing data. On the other hand, the jobs descriptions are realistic data gathered from LinkedIn and Monster websites.

\section{A. An Illustrative Example}

This example uses a simple job's profile with set of features. Minimal features were included in this example to simplify the concepts. Additionally, we use a diversity pool for alternatives specialties.

A design goal of MCAIS-JRS is to recommend the recognized antigens that represent the qualified applicants to the current job position. We will take the following job's profile: Job code $=2$, MSC degree with 4.0 GPA and spatiality in Electrical Engineering, BSC degree with 4.0 GPA in Electrical Engineering, English language with excellent level, OO programming skills, and 8 experience years. Figure 4 presents the diversity pool of the specialties alternatives for this job.

\begin{tabular}{cc}
\hline Sp-Code & Name \\
\hline 1 & Computer Science \\
\hline 2 & Electrical Engineering \\
\hline 3 & Telecommunication \\
\hline 4 & Business Administration \\
\hline
\end{tabular}

Fig. 4. The diversity pool of specialties alternatives for the current job

The algorithm will produce the following antibodies for all features with their alternatives of the current job's profile. 


\begin{tabular}{cccc}
\hline $\begin{array}{c}\text { Ab } \\
\text { code }\end{array}$ & $\begin{array}{c}\text { Feature } \\
\text { value }\end{array}$ & $\begin{array}{c}\text { Feature } \\
\text { strength }\end{array}$ & $\begin{array}{c}\text { Feature } \\
\text { type }\end{array}$ \\
\hline 1 & 1 & 8 & <MSC DEGREE> \\
\hline 2 & 4.0 & 5 & <MSC GPA> \\
\hline 3 & 2 & 5 & <MSC SPECIALTY > \\
\hline 4 & 1 & 10 & <BSC DEGREE> \\
\hline 5 & 4.0 & 7 & <BSC GPA> \\
\hline 6 & 2 & 8 & < BSC SPECIALTY > \\
\hline 7 & 1 & 10 & <LANGUAGE> \\
\hline 8 & 3 & 3 & <LANGUAGE \\
\hline 9 & 3 & 1 & LEVEL> \\
\hline 10 & 8 & 7 & <EXPERIENCE> \\
\hline 11 & 1 & 0 & Specialty alternative 1 \\
\hline 12 & 3 & 3 & Specialty alternative 2 \\
\hline 13 & 4 & 2 & Specialty alternative 3 \\
\hline
\end{tabular}

Fig. 5. Antibodies pool of the current job,

Then, the algorithm proceeds to compute the distance between the antibodies and the current antigen. The following ranked list of applicants (antigens) with their affinities for the current job will be produced as Fig. 6 .

Note that the parameters in Fig. $6, A_{1}, A_{4}, A_{7}$, and $A_{10}$ denote the $\mathrm{PhD}, \mathrm{MSc}, \mathrm{BSC}$, and Diploma degrees respectively. They have 1 if the applicant has the degree or 0 if not. $A_{2}, A_{5}, A_{8}$, and $A_{11}$, represent the GPA for the different degrees. $A_{3}, A_{6}, A_{9}$, and $A_{12}$, denote the specialty of the different degrees and the values 1,2,3, and 4 in these columns represent Computer Science, Electrical Engineering, Telecommunication, and Business Administration. $\mathrm{A}_{13}$ and $\mathrm{A}_{14}$ used for the language and its level. In $A_{13}$, the value 1 represents the English language while 1, 2, and 3 in $\mathrm{A}_{14}$ column represent the low, medium, and excellent level respectively. Finally, $\mathrm{A}_{15}$ and $\mathrm{A}_{16}$ denote the skill and the experience. The different values in $\mathrm{A}_{15}$ represent the different skill types.

\begin{tabular}{ccccccccccccccccccc}
\hline$A_{9}$ & $A_{1}$ & $A_{2}$ & $A_{3}$ & $A_{4}$ & $A_{5}$ & $A_{6}$ & $A_{1}$ & $A_{8}$ & $A_{9}$ & $A_{10}$ & $A_{11}$ & $A_{12}$ & $A_{13}$ & $A_{14}$ & $A_{15}$ & $A_{16}$ & Test & Affinity \\
\hline 0 & 0 & 0 & 0 & 1 & 4.1 & 2 & 1 & 4.0 & 2 & 0 & 0 & 0 & 1 & 2 & 3 & 9 & $\nabla$ & 0.022381 \\
\hline 8 & 1 & 4.0 & 2 & 1 & 4.0 & 2 & 1 & 4.0 & 2 & 0 & 0 & 0 & 1 & 3 & 5 & 12 & $\nabla$ & 0.0714286 \\
\hline 2 & 1 & 4.5 & 2 & 1 & 4.3 & 3 & 1 & 4.9 & 2 & 0 & 0 & 0 & 1 & 2 & 6 & 11 & $\nabla$ & 0.0780952 \\
\hline 4 & 0 & 0 & 0 & 1 & 4.0 & 2 & 1 & 3.5 & 2 & 1 & 3.0 & 3 & 1 & 3 & 6 & 12 & $\nabla$ & 0.0785714 \\
\hline 7 & 1 & 4.5 & 1 & 1 & 4.0 & 3 & 1 & 4.9 & 2 & 0 & 0 & 0 & 1 & 1 & 9 & 7 & $\nabla$ & 0.115119 \\
\hline 3 & 1 & 4.0 & 2 & 1 & 4.0 & 2 & 1 & 4.0 & 2 & 0 & 0 & 0 & 1 & 1 & 5 & 10 & $\nabla$ & 0.1190476 \\
\hline 5 & 0 & 0 & 0 & 1 & 3.8 & 4 & 1 & 4.0 & 2 & 0 & 0 & 0 & 1 & 3 & 6 & 3 & $\nabla$ & 0.1193571 \\
\hline 9 & 1 & 4.0 & 2 & 1 & 4.0 & 2 & 1 & 4.0 & 2 & 1 & 3.8 & 2 & 1 & 1 & 7 & 7 & $\nabla$ & 0.1279762 \\
\hline 6 & 0 & 0 & 0 & 0 & 0 & 0 & 1 & 3.5 & 3 & 1 & 3.1 & 1 & 1 & 2 & 8 & 12 & $\nabla$ & 0.3166667 \\
\hline 1 & 0 & 0 & 0 & 0 & 0 & 0 & 1 & 3.0 & 4 & 1 & 3.0 & 2 & 1 & 1 & 5 & 19 & $\nabla$ & 0.347619 \\
\hline
\end{tabular}

Fig. 6. The ranked list of applicants (antigens) with their affinities for the current job

\section{B. Experimental Evaluation}

For algorithm's evaluation, we applied the accuracy metrics that empirically measures how well a system can predict the most appropriate applicants for a specific job. For each posted job, there is a specific category that the active job related to it. Then, the algorithm generates recommendations using the similarity measures between job's features and applicants' profiles. If the algorithm able correctly to predict applicants who have the same job's category of the active job, then the algorithm is considered to perform well. However, for each job a ranked list of applicants is produced and evaluated such that whether those selected applicants match the same category for the active job. The accuracy metrics will be used to measure the frequency with which a recommender system makes correct or incorrect decisions about whether the selected applicant is appropriate.

In the experiment, we produce the recommended list of applicants that selected by MCAIS-JRS, then the result is evaluated and compared with our previous AIS algorithm [3]. In both algorithms, we check whether the applicants in the recommendation list from the same category of the active job. We applied the precision, recall, and F1 measures as details in the following paragraphs. The precision defined as the ratio of relevant items selected to number of items selected [23]. In our algorithms, it represents the ratio of relevant applicants selected to number of applicants selected.

$$
\mathrm{P}=\frac{\mathrm{N}_{\mathrm{rs}}}{\mathrm{Ns}}
$$

where $N_{r s}$ refers to the number of relevant selected applicants and $N_{s}$ refers to number of selected applicants. The recall defined as the ratio of relevant items selected to the total number of relevant items available [23]. In our algorithms, it represents the ratio of relevant applicants selected to the total number of relevant applicants. It denotes the probability that a relevant applicant will be selected.

$$
\mathrm{R}=\frac{\mathrm{N}_{\mathrm{rS}}}{\mathrm{Nr}}
$$

where $N_{r}$ refers to the number of relevant applicants. Several approaches have been taken to combine precision and recall into a single metric. The $F 1$ metric that combines precision and recall into a single number has been used to evaluate recommender systems in [24].

$$
\mathrm{R}=\frac{\mathrm{N}_{\mathrm{rS}}}{\mathrm{Nr}}
$$

We perform the experiments according to different levels of similarity thresholds. The threshold levels determine which applicants will be included in the selected set of applicants depending on the affinity between the job's profile and the applicant. The experiments show that the MCAIS-JRS accuracy is high and more likely the accuracy of the traditional AIS. They show high accuracy when the threshold decreased. See Figures 7, 8, and .9, for precision, recall, and F1 respectively. 


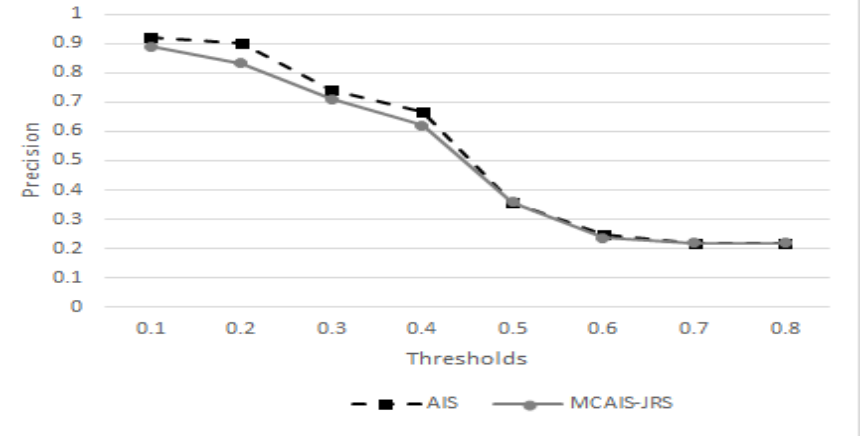

Fig. 7. The Precision rate of MCAIS-JRS and traditional AIS

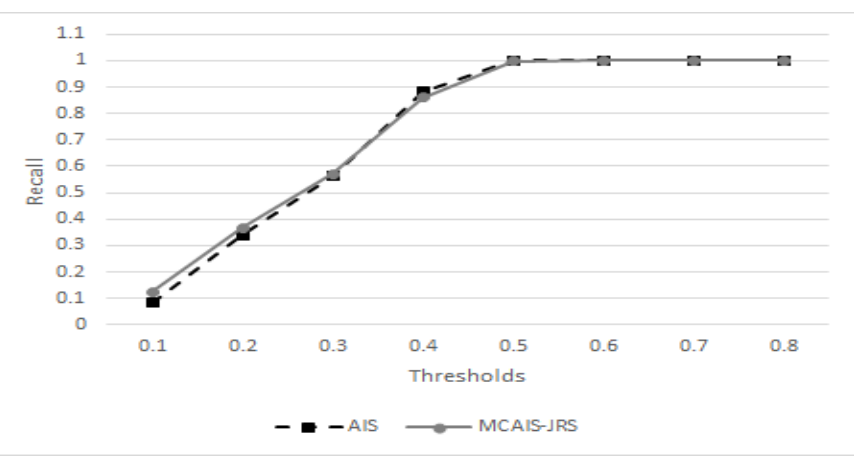

Fig. 8. The Recall rate of MCAIS-JRS and traditional AIS

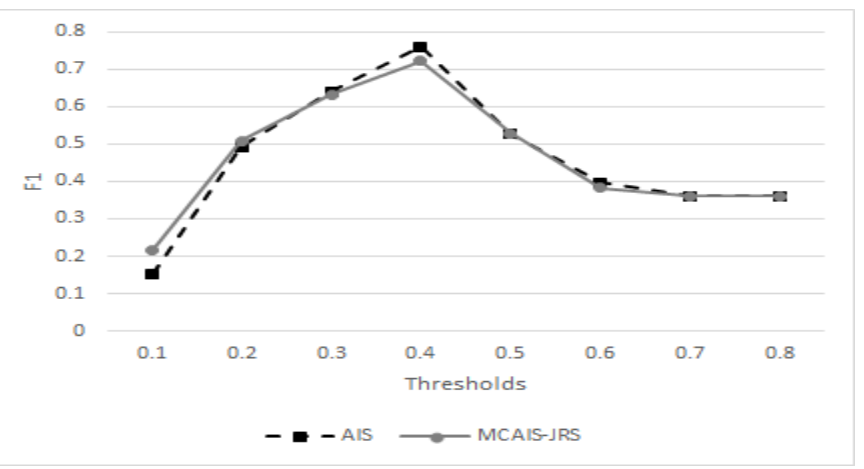

Fig. 9. The F1 rate of MCAIS-JRS and traditional AIS

\section{Scalability Test}

Scalability is an extremely important issue of recommender system efficiency, especially when they are used in larger datasets. We evaluate the scalability of the proposed algorithm by varying the datasets sizes. However, the execution time of the MCAIS-JRS and traditional AIS algorithms are compared using different datasets sizes. As seen in the previous section, both algorithms give relatively the likewise accuracy rates using different accuracy measures but we need to examine the system's efficiency when the datasets size grow. Figure 10 shows a comparison between MCAIS-JRS and the traditional AIS based on the execution time with different datasets sizes. When the number of applicants increased in the system, the execution time increases in a linear fashion, indicating that the traditional AIS efficiency is highly reduced while the execution time is stable in MCAIS-JRS and the system efficiency remains high in different datasets sizes. The maximum execution time for traditional AIS is 718 seconds with 7000 applicants in the system, while the MCAIS-JRS takes between 84 and 163 seconds in different datasets sizes. Specifically, the MCAISJRS achieves high efficiency with reasonable performance.

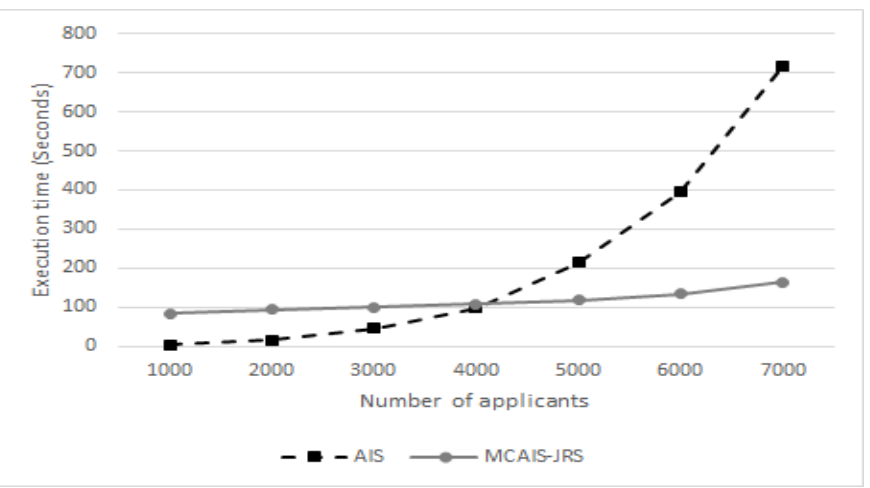

Fig. 10. Execution time of MCAIS-JRS and traditional AIS with different datasets sizes

\section{CONCLUSION}

The discovery of monoclonal antibodies has encouraged a revolution in medicine that is probably only second to the discovery of vaccination. In this technology, the antibodies were produced in laboratories from single antibody cell. Our proposed model represents an optimization technique based on the abstractions of the monoclonal antibodies production principle and the AIS processes, and they are utilized towards enhancing the job recommendation. Hence, the job's requirements and applicant's resume exploited and used in the implementation of MCAIS-JRS that recommends the most qualified applicants to a specific job. In this algorithm, we produce antibodies using the monoclonal antibodies production principle by treating each job's feature as a single antibody. Additionally, we perform the diversity of job's requirements using diversity operator instead of mutation strategy that produces antibodies limited by the number of job's features and their alternatives. Moreover, as presented in experimental results section, our new proposed algorithm produces a good recommendation result and enhances the system efficiency compared with our previous AIS model.

\section{ACKNOWLEDGMENT}

This work was supported by Deanship of Scientific Research and Research Center of College of Computer and Information Sciences, King Saud University. The authors are grateful for this support.

\section{REFERENCES}

[1] F. Färber, T. Weitzel and T. Keim, "An automated recommendation approach to personnel selection," in Americas Conference on Information Systems, Tampa, Florida, USA, 2003.

[2] X. Yi, J. Allan and W. B. Croft, "Matching resumes and jobs based on relevance models," in Proceedings of SIGIR, New York, NY, USA, 2007.

[3] S. T. Al-Otaibi and M. Ykhlef, "An artificial immune system for job recommendation," in proceedings of 3rd IEEE International Conference and Workshop on Bioinspired Intelligence (IWOBI 2014), Universidad Nacional, Liberia,Costa Rica, 2014.

[4] M. Leenaars and C. F. M. Hendriksen, "Critical steps in the production of polyclonal and monoclonal antibodies: evaluation and recommendations," ILAR Journal, pp. Vol. 46(3) ,pp. 269-279, 2005. 
[5] S. T. Al-Otaibi and M. Ykhlef, "Survey of job recommender systems," International Journal of the Physical Sciences, pp. Vol. 7(29), pp. 51275142,2012

[6] S. T. Zheng, W. X. Hong, N. Zhang and F. Yang, "Job recommender systems: a survey," in Proceedings of the 7th International Conference on Computer Science \& Education (ICCSE 2012) , Melbourne, Australia, 2012.

[7] J. Malinowski, T. Weitzel and T. Keim, "Decision support for team staffing: an automated relational recommendation approach," Decision Support Systems, pp. [45], 3, 429-447, 2008.

[8] J. Malinowski, T. Keim, O. Wendt and T. Weitzel, "Matching people and jobs: a bilateral recommendation approach," in Proceedings of the 39th Annual Hawaii International Conference on System Sciences, Hawaii, USA, 2006.

[9] T. Keim, "Extending the applicability of recommender systems:a multilayer framework for matching human resources," in Proceedings of the 40th Annual Hawaii International Conference on System Sciences (HICSS'07), Hawaii, USA, 2007.

[10] P.-C. Chen, "A Fuzzy multiple criteria decision making model in employee recruitment," IJCSNS International Journal of Computer Science and Network Security, pp. [9], 7, 113-117, 2009.

[11] K. Kowsari, M. Yammahi, N. Bari, R. Vichr, F. Alsaby and S. Y. Berkovich, "Construction of FuzzyFind Dictionary using Golay Coding Transformation for Searching Applications" International Journal of Advanced Computer Science and Applications(IJACSA), 6(3), 2015.

[12] M. Fazel-Zarandi and M. S. Fox, "Semantic matchmaking for job recruitment: an ontolgy based hybrid approach.," in In Proceedings of the 3rd International Workshop on Service Matchmaking and Resource Retrieval in the Semantic Web at the 8th International Semantic Web Conference, Washington D.C., USA, 2010.

[13] A. Singh, R. Catherine and K. Visweswariah, "PROSPECT: A System for screening candidates for recruitment," in Proceedings of 19th ACM International Conference on Information and Knowledge Management (CIKM'10), Toronto, Ontario, Canada, 2010.
[14] I. Paparrizos, B. B. Cambazoglu and A. Gionis, "Machine learned job recommendation," in proceedings of the fifth ACM conference on Recommender systems,RecSys'11, Chicago, Illinois, USA, 2011.

[15] M. Rodriguez, C. Posse and E. Zhang, "Multiple objective optimization in recommender systems," in Proceedings of the sixth ACM conference on Recommender systems, New York, NY, USA, 2012.

[16] Y. Lu, S. E. Helou and D. Gillet, "A recommender system for job seeking and recruiting websites," in International World Wide Web Conference Committee (IW3C2), Rio de Janeiro, Brazil., 2013.

[17] A. Gupta and D. Garg, "Applying data mining techniques in job recommender system for considering candidate job preferences," in Proceedings of the International Conference on Advances in Computing,Communications and Informatics (ICACCI), Delhi, India, 2014.

[18] D. Dasgupta, "Advances in artificial immune systems," IEEE Computational Intelligence Magazine, pp. 40-49, 2006.

[19] L. d. Castro and J. Timmis, "Artificial immune systems: a novel paradigm for pattern recognition," Artificial Neural Networks in Pattern Recognition , Alonso L, Corchado J,Fyfe C (eds), p. pp. 67-84. University of Paisley, 2002.

[20] R. A. Goldsby, T. J. Kindt and B. A. Osborne, Kuby Immunology, Fifth Edition, W. H. Freeman, 2002.

[21] U. Aickelin and D. Dasgupta, "Artificial Immune Systems," in Search Methodologies: Introductory Tutorials in optimization and decision support techniques, Springer, 2003, pp. 375-399.

[22] G. Kohler and C. Milstein, "Continuous cultures of fused cells secreting antibody of predefined specificity," Nature, pp. Vol. 256, pp. 495 - 497, 1975.

[23] J. A. Herlocker, J. A. Konstan, L. G. Terveeni and J. T. Riedl, "Evaluating collaborative filtering," ACM Transaction, 2004.

[24] B. Sarwar, G. Karypis, J. Konstan and J. Riedl, "Incremental SVDBased algorithms for highly scaleable recommender systems," in Proceedings of the 5th International Conference on Computer and Information Technology (ICCIT '02), 2002. 